\title{
NUMERICAL SIMULATION OF COLLOIDAL DISPERSION FILTRATION: DESCRIPTION OF CRITICAL FLUX AND COMPARISON WITH EXPERIMENTAL RESULTS
}

\author{
Patrice Bacchin $^{1}$, Benjamin Espinasse ${ }^{1}$, Yolaine Bessiere ${ }^{1}$, David F. Fletcher ${ }^{2}$, Pierre Aimar $^{1}$ \\ ${ }^{1}$ Laboratoire de Génie Chimique, Université Paul Sabatier, 118 Route de Narbonne, 31062 Toulouse, \\ France \\ ${ }^{2}$ Department of Chemical Engineering, University of Sydney, NSW 2006, Australia
}

\begin{abstract}
Introduction
Colloidal dispersions are encountered in many fluids requiring filtration via membrane processes. However, these dispersions exhibit special properties, mainly because of the surface interactions (repulsive and attractive) occurring between the colloid particles. Such interactions have been shown to be responsible for aggregation phenomena on the membrane surface induced by the permeate flux; thus leading to the concept of critical flux which separates filtration conditions below which mass accumulation is reversible (concentration polarization) and above which it is irreversible (coagulated deposit). The aim of this work is to combine a representation of the physico-chemical properties of these colloidal dispersions with a rigorous simulation of momentum and mass transfer using CFD modelling. The ultimate aim is to describe the first appearance of a deposit on the membrane, to compare this simulated value with experimental determinations of critical flux.
\end{abstract}

\section{Mathematical formulation and membrane transfer model}

The mathematical model is based on the same formulation as that developed by Wiley and Fletcher [1] and described in detail in [2]. Conservation equations for the conservation of mass, momentum and particle mass fraction are solved assuming incompressible fluids and no slip between the particles and the water. The membrane is assumed to be perfectly rejecting. The fluid extraction via the membrane (local permeate flux) is modeled via

$v_{w}=\frac{1}{\mu R_{m}}(\Delta P-\Delta \pi)=\frac{1}{\mu R_{m}}\left(\Delta P-\pi_{m}\right)$

(Equation 1)

where $\mathrm{R}_{\mathrm{m}}$ is the membrane resistance, and $\Delta \mathrm{P}$ and $\Delta \pi$ are the local differences of the applied pressure and the osmotic pressure across the membrane, respectively. If the membrane is fully retentive the difference in osmotic pressure is the osmotic pressure at the membrane wall, $\pi_{\mathrm{m}}$.

\section{Model for colloidal transport properties}

The behavior of the colloidal suspension is based on the variation of the osmotic pressure with the particle volume fraction. The colloidal osmotic pressure (or more precisely the variation of the osmotic pressure with the volume fraction) has been theoretically shown as useful to predict the behavior of concentrated colloidal dispersion and phase transitions occurring in the dispersion [3]. The critical transition between the dispersed phase and the aggregated phase (defined in [4] as a spinodal decomposition) is modeled by a zero derivative of osmotic pressure with volume fraction. A critical volume fraction (and an associated critical osmotic pressure) can be defined above which an irreversible aggregation occurs between particles.

\section{Description of the colloidal osmotic pressure}

Experimental measurements of the colloidal osmotic pressure have been performed on a stable latex dispersion (120 nm in diameter with a standard deviation of $20 \mathrm{~nm}$ ) with an osmotic stress technique [5]. This technique is based on the compression of the dispersion put in a dialysis bag with a stressing polymer. At equilibrium, the chemical potential of water on either side of the membrane is equal and therefore the osmotic pressure of the dispersion equals that of the polymer in the reservoir. Increasing the amount of polymer in the reservoir (and the associated osmotic pressure) and measuring the concentration of the dispersion (determined with a drying mass balance Mettler-Toledo, Switzerland at $120^{\circ} \mathrm{C}$ ) when 
equilibrium is reached allows determination of the variation of colloidal osmotic pressure with the volume fraction (symbols in Figure 1). Qualitative reversibility tests were carried out on the equilibrated latex dispersion by immersing them in an excess of water to see whether they could be dispersed. In Figure 1, black symbols represent latex dispersion for which the concentration by osmotic stress was impossible (aggregated phase). A phase transition occurs at a volume fraction around 0.58.

In this work, we fit the experimental variation of osmotic pressure for latex suspension with a mathematical relationship having a zero derivative at the critical transition. The relationship used for this work (for volume fractions below the critical value) has the following form:

$\pi=e^{\left(\frac{1}{\frac{1}{a \phi+b}+\frac{1}{c \phi+d}+\frac{1}{e \phi+f}}\right)}$

(Equation 2)

with values for the fitting parameters as given in Table 1 for the osmotic pressure in Pa.

Table 1: Values of Parameters of Equation 2 Fitting the Experimental Data of Figure 1.

\begin{tabular}{|l|l|}
\hline Parameter & Value \\
\hline $\mathrm{a}$ & 11.82225446 \\
\hline $\mathrm{b}$ & 2.859472081 \\
\hline $\mathrm{c}$ & -475.6715757 \\
\hline $\mathrm{d}$ & -2127.791606 \\
\hline $\mathrm{e}$ & -44622.80005 \\
\hline $\mathrm{f}$ & 26320.00625 \\
\hline
\end{tabular}

This expression has a maximum osmotic pressure with volume fraction (the spinodal decomposition) at a volume fraction of 0.5765 , with an associated critical osmotic pressure of $14158 \mathrm{~Pa}$.

Above the critical volume fraction, the variation of the osmotic pressure with volume fraction has been fitted with the relationship:

$$
\pi=\pi_{\text {crit }}\left(\left[\frac{\phi_{\text {cp }}-\phi_{\text {crit }}}{\phi_{\text {cp }}-\phi}\right]^{\mathrm{m}}-\mathrm{m} \frac{\phi-\phi_{\text {crit }}}{\phi_{\mathrm{cp}}-\phi_{c r i t}}\right)
$$

This equation is a modified form of the classical form for the compressive yield stress compression [6] which ensures that at the critical point the osmotic pressure and its derivative are continuous with volume fraction. This relationship has an infinite limit at the close packed volume fraction. The exponent, $\mathrm{m}$, in this relation corresponds to the compressibility of the condensed phase: a small value of $\mathrm{m}$ gives a small compressibility i.e. the solid pressure increases rapidly with volume fraction. To fit the experimental data, the compressibility $\mathrm{m}$ is set to 5.7 . 


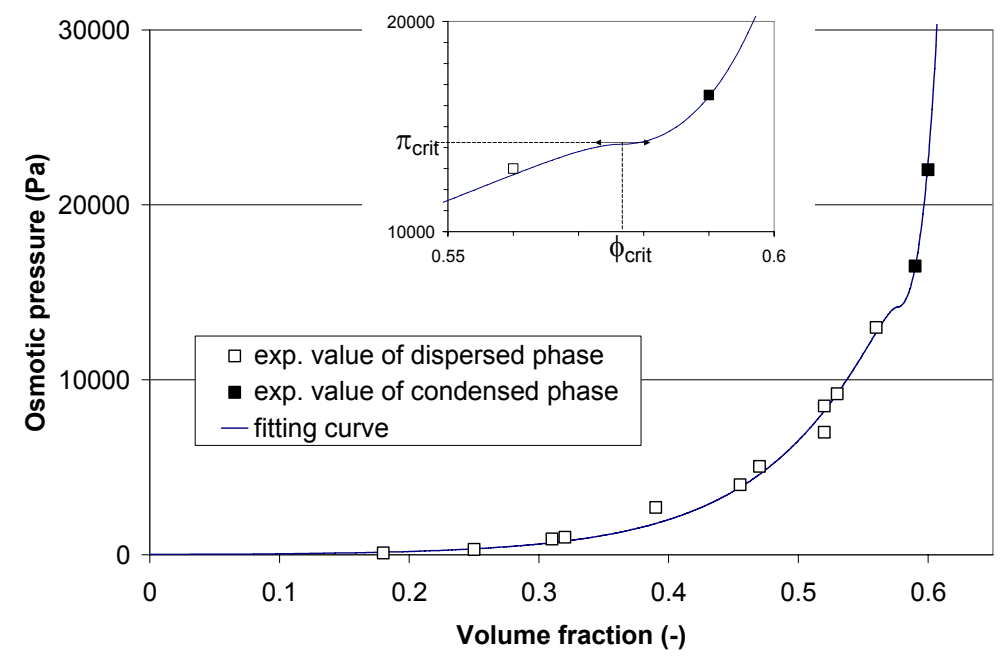

Figure 1: Variation of osmotic pressure with volume fraction. The symbols represent the experimental osmotic pressure value for the latex dispersion (open symbol: latex in dispersed phase, closed symbol: latex in condensed phase). The line represents the model fit.

\section{Consequences of colloidal osmotic pressure on transfer phenomena}

The variation of osmotic pressure has two major consequences on transfer phenomena:

1. it affects the permeate flux in the classical manner given in equation (1);

2. it affects the way mass accumulates. Here we model collective diffusion [7] via a Stokes Einstein law where the diffusion coefficient is proportional to the derivative of the osmotic pressure with respect to the volume fraction:

$$
D(\phi)=\frac{V_{p}}{6 \pi \mu a H(\phi)} \frac{d \pi}{d \phi}
$$

where $a$ is the particle radius and $\mathrm{V}_{\mathrm{p}}$ its volume. The Happel function $H(\phi)$ accounts for the effect of concentration on the drag force and is given by:

$$
H(\phi)=\frac{6+4 \phi^{\frac{5}{3}}}{6-9 \phi^{\frac{1}{3}}+9 \phi^{\frac{5}{3}}-6 \phi^{2}}
$$

These expression leads to the variation of the diffusion coefficient with volume fraction presented in Figure 2. In such modeling, repulsive long distance interactions induce an increase of osmotic pressure and a relative increase in the diffusion coefficient. When attraction between particles becomes important (as the critical transition is approached), it leads to a decrease in the osmotic pressure variation with volume fraction and an associated decrease in diffusion: the spinodal decomposition gives a coefficient of diffusion of zero, causing irreversible phenomena. Above the critical volume fraction, the diffusion of the condensed phase is given by the derivative of Equation 3 with respect to the volume fraction:

$$
\frac{d \pi}{d \phi}=\pi_{\text {crit }}\left(\frac{m}{\phi_{\text {cp }}-\phi}\left[\frac{\phi_{\mathrm{cp}}-\phi_{\text {crit }}}{\phi_{\mathrm{cp}}-\phi}\right]^{\mathrm{m}}-\frac{m}{\phi_{\mathrm{cp}}-\phi_{\text {crit }}}\right)
$$


The diffusion of the condensed phase used in this work is similar to the cake diffusivity developed by Sherwood [8] for description of compressible filter-cake compaction.

The variation of the viscosity with volume fraction is accounted for using the Eilers-Chong relationship [8]:

$$
\frac{\mu(\phi)}{\mu_{b}}=\left[1+\frac{1.25 \phi}{1-\phi / \phi_{c p}}\right]^{2}
$$

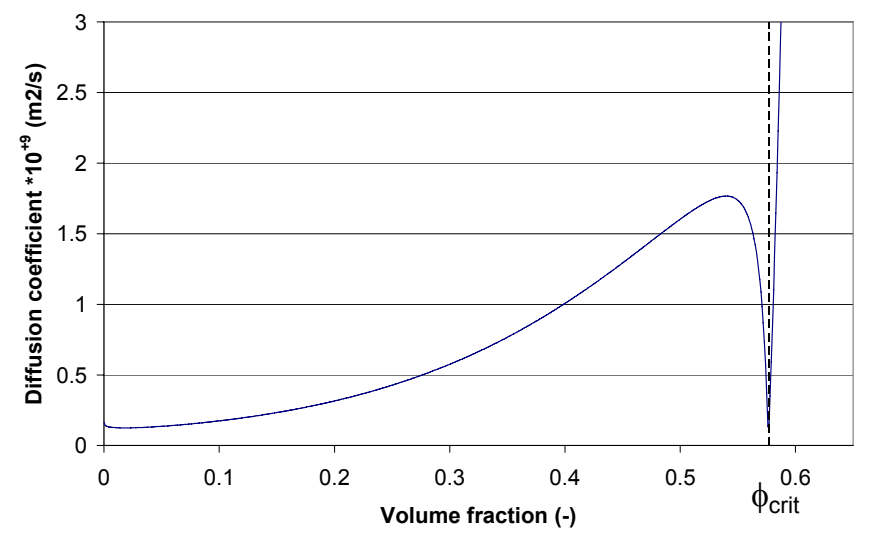

Figure 2: Variation of the diffusion coefficient with the volume fraction for the osmotic pressure model presented in Figure 1. The critical transition is depicted with a diffusion coefficient of zero.

\section{Solvent transfer and colloidal osmotic pressure}

As shown in Equation 1, a classical filtration law is used to link the permeate flux to the colloidal osmotic pressure at the membrane surface. Such a model is classical when considering the polarization concentration mechanism. However, here the same law is used to describe the filtration when the critical volume fraction is reached (i.e. when a condensed phase (gel or deposit) appears at the interface). Such a description is based on the analogy made between the osmotic pressure model and the gel layer model described by [10] and on the relationship between the osmotic pressure (which above the critical point can be seen as the stress within the matrix of particles) and the liquid pressure. It can be assumed [8] that the sum of the liquid pressure and the osmotic pressure is constant in the condensed phase (Figure 3):

$$
p_{i}+\pi_{i}=p_{m}+\pi_{m}
$$

By applying Darcy's law through the condensed phase having a resistance, $\mathrm{R}_{\mathrm{c}}$ :

$$
v_{w}=\frac{p_{i}-p_{m}}{\mu R_{c}}
$$

Assuming, when a condensed phase forms at the membrane, the osmotic pressure at the interface $\pi_{\mathrm{i}}$ is equal to $\pi_{\text {crit }}$ (the maximum reachable osmotic pressure in the dispersed phase), the combination of equations 1 and 9 leads to the following expression for the osmotic pressure of the condensed phase at the membrane:

$$
\pi_{m}=\pi_{c r i t}+J \mu R_{c}
$$

When the critical point is reached and a condensed phase is formed, the osmotic pressure used in equation 1 can then be viewed as the addition of a critical osmotic pressure, $\pi_{c r i t}$, and a contribution 
related to a cake resistance (the second term in equation 10). Equation 1 for solvent transfer can then be used to understand the formation and the growth of deposit (or gel) layers.

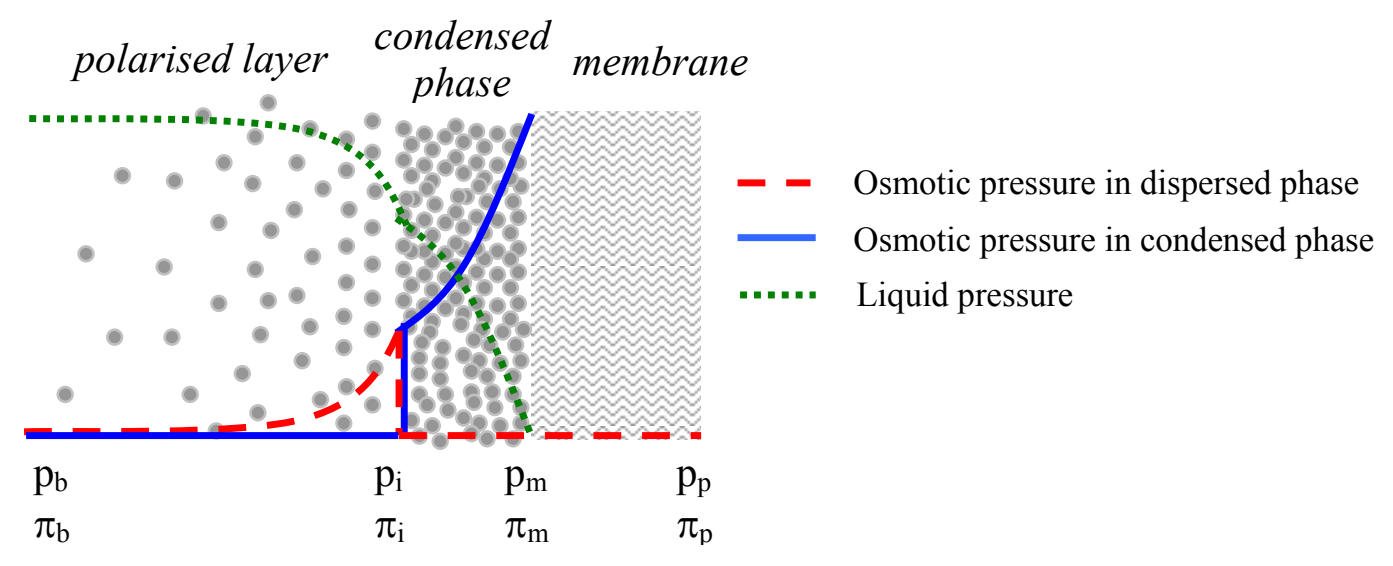

Figure 3: Evolution of the liquid pressure and the osmotic pressure through the polarized layer and the condensed phase on a membrane (subscript $\mathrm{b}$ for bulk, $\mathrm{i}$ for interface, $\mathrm{m}$ for membrane and $\mathrm{p}$ for permeate).

\section{Numerical Method and Solution Procedure}

The equations for mass and momentum conservation were solved using the finite volume CFD code ANSYS CFX5, (version 5.7). A two dimensional, axisymmetric slice of the tube was meshed with a structured mesh, generated in ICEM- HEXA, having a very fine mesh near the membrane surface. The nearest node from the surface was located one micron away from the surface and the mesh expanded smoothly towards the tube axis. Wall extraction was implemented via user routines and the expressions for the variable viscosity, diffusivity and osmotic pressure were written in CFX Expression Language. This made the model very flexible and easy to use for the study of parameter variations and model sensitivities.

At low extraction rates, convergence to a steady-state solution was very easy, with low normalized residuals $\left(<10^{-5}\right)$ and good mass balances $(<0.01 \%)$ being obtained in a few hundred iterations. At higher extraction rates convergence was more difficult because of the strong coupling between the particle volume fraction and the fluid properties. Performing under-relaxation using Expert Parameters proved to be very helpful. Once the particle fraction approached the critical level for deposit formation it was found to be optimal to switch to a transient solution. A pressure step of typically 0.05 bars was made and a transient simulation was run until this reached a new steady-state.

The model results are extremely sensitive to the numerical mesh and the accuracy of the numerical scheme. Whilst first order differencing gave much faster convergence, the results showed enormous differences from a higher order scheme. Using the High Resolution scheme, which seeks to use an optimal combination of first and second order schemes to provide a bounded solution proved optimal, as pure second order scheme led to overshoots. In addition, it was found to be beneficial to ramp down the extraction flux at the end of the membrane to avoid a step change. This was done using a hyperbolic tangent function over the last $0.05 \mathrm{~m}$ of the membrane.

\section{Results}

\section{Critical flux description}

The use of the modeling of concentrated colloidal phases presented above in the CFD code provides a realistic description of mass accumulation at the membrane. It is then possible to simulate the formation of the condensed phase from the polarized layer. This irreversible (and then critical) transition between a dispersed and a condensed accumulation can be numerically found when the driving pressure is large 
enough. However, this driving pressure is different if the membrane permeability it changed but the transition occurs for the same permeate flux; it is defined as the critical flux.

The system studied comprised a $1.4 \mathrm{~m}$ long, $6 \mathrm{~mm}$ diameter tube, comprising a $0.1 \mathrm{~m}$ entry length (to develop the flow), a $1.2 \mathrm{~m}$ member section and a $0.1 \mathrm{~m}$ outlet zone. A particle laden flow containing 0.5 $\mathrm{g} / 1$ of $120 \mathrm{~nm}$ diameter particles entered the tube with a mean liquid velocity of $0.6 \mathrm{~m} / \mathrm{s}$ in cross-flow mode. The membrane resistance permeability was $5.9 \times 10^{-12} \mathrm{~m}^{-1}$. Figure 4 shows the variation with driving pressure of both the maximum (at the outlet of membrane channel) and mean values of the particle volume fraction on the membrane surface. It is evident that the critical conditions are reached at the end of membrane for a driving pressure of around 2.6 bars. This corresponds to a flux of around $0.04 \mathrm{~kg} \mathrm{~m}^{-2} \mathrm{~s}^{-1}$, as shown in Figure 5. For these conditions, the maximum osmotic pressure of the dispersed phase (around 0.14 bar in Figure 1) represents only 5\% of the applied pressure. The effect of the osmotic pressure on the permeate flux (Equation 1) is then negligible (Figure 5). A deviation from linearity just appears when the condensed phase begins to form at the end of the membrane channel: the code describes a strong form of critical flux (permeate flux is equal to water flux below the critical value).

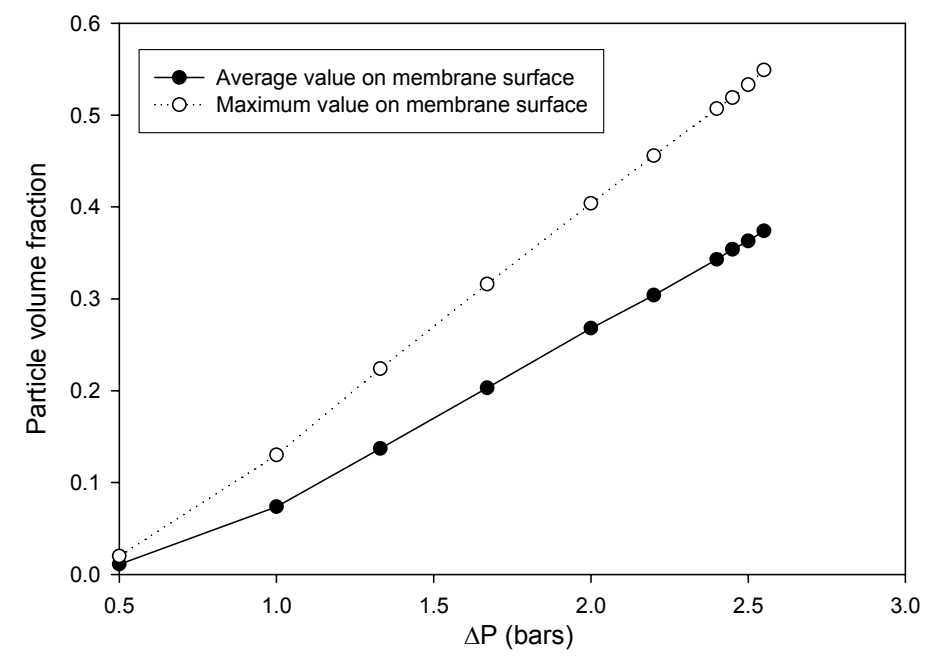

Figure 4: The calculated average and maximum particle fraction on the membrane surface as a function of the trans-membrane pressure.

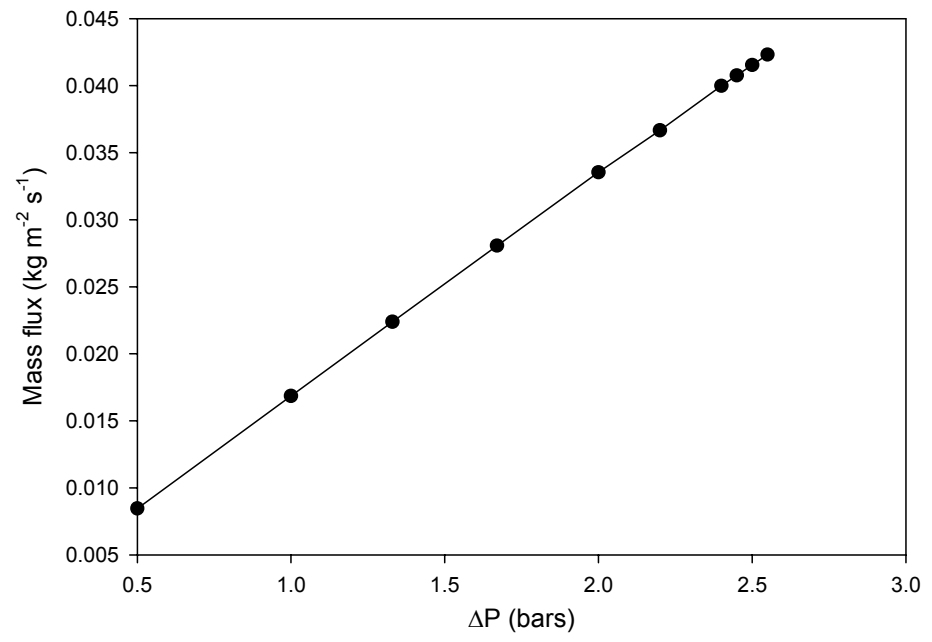

Figure 5: The calculated flux as a function of the trans-membrane pressure. 
Comparison with experimental value

Experimental filtrations have been performed for the same hydrodynamic conditions $(0.6 \mathrm{~m} / \mathrm{s})$ and with latex particles dispersed in $0.001 \mathrm{M} \mathrm{KCl}$ characterized by the osmotic pressure variation with volume fraction presented in Figure 1 [5]. The filtration allows an accurate determination of the critical flux according to a procedure based on controlled alternating increasing and decreasing pressure steps with the measurements of the steady state flux. The experimental critical flux has been determined at $10.1 \times 10^{-6} \mathrm{~m} / \mathrm{s}$ [11]. The simulated values of critical flux are then approximately 4 times higher than that observed experimentally. Such a discrepancy could be explained by the heterogeneity of the porous wall which affect locally the permeate flux [12]. For a given pressure and then an averaged permeate flux, critical conditions can exist at the membrane surface with higher local permeate flux and then more important particle accumulation [11]. Future work will investigate the effect of such distributions on the mass accumulation in a membrane channel.

\section{Conclusions}

A rigorous simulation of momentum and mass transfer using CFD modelling has been developed to describe colloids accumulation at a membrane surface during cross flow filtration. These simulations integrate detailed modeling of physico-chemical properties specific to colloidal dispersions (because of the surface interactions (repulsive and attractive) occurring between the colloids particles). These interactions are accounted for via the experimental variation of the colloidal osmotic pressure with volume fraction which is fitted by a relationship integrated into the CFD code. It contains a description of the colloidal phase transition leading to the formation of a condensed phase (deposit or gel layer) from the accumulated dispersed phase (concentration polarization) and then to determination of the critical flux which separates filtration conditions below which mass accumulation is reversible (in dispersed phase) and above which it is irreversible (in condensed phase). The computed value of critical flux is compared with that determined experimentally for a dispersion of latex particles.

\section{Acknowledgements}

We thank Dr Alan Burns of ANSYS CFX for providing the original wall extraction model and assistance with implementation of the model.

\section{References}

[1] Wiley, D.E. and D.F. Fletcher, "Computational Fluid Dynamics Modelling of Flow and Permeation for Pressure-driven Membrane Processes", Desalination, 145 (2002) 183-186.

[2] Fletcher, D.F. and D.E. Wiley, "A Computational Fluid Dynamics Study of Buoyancy Effects in Reverse Osmosis", J. Membr. Sci., 245 (2004) 175-181.

[3] Jönsson, A.S., B. Jönsson, Ultrafiltration of Colloidal Dispersions - A Theoretical Model of the Concentration Polarization Phenomena, J. Colloid Interface Sci., 180 (1996) 504-518.

[4] Russel, W.B., D.A. Saville, and W.R. Schowalter, Colloidal Dispersions, Cambridge University Press, Cambridge, (1991).

[5] Espinasse, B. Approche Théorique et Expérimentale de la Filtration Tangentielle de Colloïdes: Flux Critique et Colmatage, Thèse de l'Université Paul Sabatier, Toulouse, France, 2003.

[6] Landman K.A. and L.R. White, "Determination of the Hindered Settling Factor for Flocculated Suspensions", AIChE J., 38 (1992) 184-192.

[7] Bacchin, P., D. Si-Hassen, V. Starov, M.J. Clifton and P. Aimar, "A Unifying Model for Concentration Polarization Gel-layer Formation and Particle Deposition in Cross-flow Membrane Filtration of Colloidal Suspensions", Chem. Eng. Sci., 57 (2002) 77-91.

[8] Sherwood, J.D., "Initial and Final Stages of Compressible Filtercake Compaction", AIChE J., 43 (1997) $1488-1493$.

[9] Kissa E. "Dispersions: Characterization, Testing and Measurement", Marcel Dekker, New York, (1999).

[10] Elimelech, M. and S. Bhattacharjee, "A Novel Approach for Modelling Concentration Polarization in Crossflow Membrane Filtration Based on the Equivalence of Osmotic Pressure Model and Filtration Theory", J. Membr. Sci., 145 (1998) 223-241 
[11] Bacchin, P., B. Espinasse and P. Aimar, "Distributions of Critical Fouling Conditions: Modelling, Experiments Analysis and Consequences for Cross Flow Membrane Filtration", J. Membr. Sci., 250, (2005) 223-234.

[12] Field R.W., D. Wu, J.A. Howell J.A. and B.B. Gupta, "Critical Flux Concept for Microfiltration Fouling", J. Membr. Sci., 100, (1995) 259-272. 